Article

\title{
Strain Experienced by Caregivers of Stroke Survivors in the Western Cape
}

\begin{abstract}
Recovery after stroke is often incomplete and many stroke survivors depend on family caregivers. The demands of caring can negatively impact quality of life of these carers. This study explores levels of strain experienced by caregivers and the variables that impact on their strain. A concurrent, mixed method, descriptive design was utilized. Fifty-seven caregivers of stroke survivors who received rehabilitation at the Western Cape Rehabilitation Centre participated in the study. The Barthel Index and Caregiver Strain Index were utilised to collect quantitative data, which was statistically analysed $(p<0.05)$. Qualitative data was collected through semistructured interviews and thematically analysed.

Fifty eight percent of caregivers were experiencing high levels of strain. The following variables significantly impacted on strain namely, caregiver loss of employment (0.04), emotional pressure (0.01), patient residual cognitive and perceptual impairments (0.01), personality changes (0.01) and physical dependency (0.0012). According to the qualitative data the burden was aggravated by financial problems, patient perceptual problems and patient personality changes. The findings indicate the need for support after discharge, ongoing community support and respite care to enable time off for caregivers.
\end{abstract}

KEY WORDS: CAREGIVER, REHABILITATION, STRAIN, STROKE.

\section{INTRODUCTION}

Stroke occurs suddenly and is unpredictable in terms of affected functions and outcomes. An array of impairments including functional, cognitive and emotional, behavioural and social can result from stroke, leading to profound changes in abilities, personality, life style and life roles. Many stroke sufferers are cared for at home by informal caregivers and these changes can cause high levels of strain for caregivers (Pierce et al 2007).

According to international literature, between $29 \%$ and $43 \%$ of caregivers experience strain related to care giving duties (Ilsei et al 2008; Rochette et al 2007; Visser-Meiley et al 2005). South African studies found similar to higher levels of strain in caregivers of stroke sufferers (Wasserman et al 2009;

\section{Corresponding Author:}

Surona Visagie

P.O. Box 40

Fraserburg

6960

E-mail: surona@telkomsa.net
Kleineibst 2007). Patients in the KwaZulu Natal study by Wasserman et al (2009) received no rehabilitation while those in the Western Cape study (Kleineibst 2007) accessed out patient rehabilitation at primary level. No South African studies that assessed caregiver strain after discharge from intensive in patient rehabilitation could be found and neither of the South African studies evaluated which variables influenced strain.

The purpose of this study was to determine if caregivers of patients who received intensive rehabilitation also experienced the high levels of strain found in other South African studies and to determine which factors affected strain. This is in accordance with the literature, which calls for further research on caregiver stain in different settings (Pierce et al 2007).

\section{METHODS}

A descriptive study that utilized a concurrent mixed methods design was conducted (Kroll et al 2005). Participants were family carers of stoke sufferers who received in patient rehabilitation at the Western Cape Rehabilitation Centre
(WCRC), a specialist rehabilitation centre, during 2006. Family carers were defined as a family member or friend who was primarily responsible for the day to day care duties in a non institutional setting (MacKenzie et al 2007).

Admission records at WCRC were perused and 228 patients were identified. Of these 171 had to be excluded from the study for the following reasons: $52 \mathrm{did}$ not require caregivers, 43 resided outside the Cape Metro health district, 28 had died, nine were discharged to care facilities and 39 could not be located. The caregivers of the remaining 57 patients participated in the study.

The Barthel Index (BI) was used to determine the patients' level of physical dependence (Uyttenboogaart et al 2005). The Care Giver Strain Index (CSI) was used to determine caregivers' levels of strain (Scherbring 2002). The CSI is a validated and reliable measure consisting of 13 questions requiring Yes/No responses. Positive responses to seven or more items indicate a high level of strain (Scherbring 2002). Two researcher-developed, structured questionnaires, one for care givers and one 
for patients were used to illicit additional information on the demographic and health status of patient and care giver, residual effects of the stroke, rehabilitation process, follow up services, training and education received and care giving duties. Semi-structured interviews were used to collect in-depth data on the experiences and feelings of caregivers with regards to rehabilitation services, the availability of community support services, and their care giving duties.

All data was collected during one session in 2009 either in the caregivers' homes, or in an office at WCRC, depending on the preference of the caregiver. The primary author collected data in English and Afrikaans. A trained research assistant conducted data collection with the Xhosa-speaking caregivers. For quantitative data, analysis of variance (ANOVA), Mann-Whitney $\mathrm{U}$ and Chi Squared tests were used to determine if there were any statistically significant relationships between CSI and dependent variables $(p<0.05)$. Transcribed qualitative data was translated into English. Transcripts were reviewed and emerging themes were identified. Where themes corresponded with quantitative data the results were integrated. Where new themes were identified they were analysed and presented with narrative evidence.

Participation in the study was voluntary and written informed consent was obtained from participants before data collection commenced. Participant information was kept confidential. The study was registered with the Committee for Human Research at Stellenbosch University. Permission to access patient folders was obtained from the director at the WCRC. The researcher arranged for reassessment of patients at WCRC where this was indicated. Caregivers who experienced strain were referred for respite care, adult day care or home based care.

\section{RESULTS}

\section{Demographic details}

As shown in table 1 the majority of caregivers $(34 ; 59 \%)$ were between 40 and 59 years of age, with a mean age of 48 years. The majority of caregivers $(51 ; 89 \%)$ were female and $22(38 \%)$ caregivers were the spouses of stroke survivors. Eleven (19\%) caregivers had to resign from employment to take on care giving duties. Having to resign from employment was the only demographic variable that had a statistically significant impact on caregiver strain $(p=0.04)$. Financial challenges were also a recurring theme in the qualitative data as expressed in the following quotation: “... no money for paraffin, sometimes we have no food" (52 years old, sister).

\section{Caregiver strain}

According to the Caregiver Strain Index (CSI) findings, $33(58 \%)$ of the caregivers experienced high levels of strain. The mean CSI score was 7.7 with four of the caregivers scoring the maximum of 13 .

\section{Unresolved effects of the stroke}

As indicated in Table 2, physical dependence had a significant impact on caregiver strain $(p<0.001)$, as did personality changes $(0.01)$, and cognitive and perceptual problems (0.01).

Caregivers expanded on personality changes in the following words: "My husband is a stranger... The children... do not know him as a father" (44 years old, wife)

Table 1: Demographic information of Stroke sufferers and caregivers

\begin{tabular}{|c|c|c|c|}
\hline \multicolumn{2}{|l|}{ Demographic characteristic } & \multirow{2}{*}{$\begin{array}{l}\text { Patients n = } 57 \\
33(58 \%)\end{array}$} & \multirow{2}{*}{$\begin{array}{l}\text { Caregivers } \mathbf{n}=57 \\
6(11 \%)\end{array}$} \\
\hline Gender & Male & & \\
\hline & Female & $24(42 \%)$ & $51(89 \%)$ \\
\hline \multirow[t]{6}{*}{ Age } & $<29$ & $4(7 \%)$ & $5(9 \%)$ \\
\hline & $30-39$ & $4(7 \%)$ & $8(14 \%)$ \\
\hline & $40-49$ & $8(14 \%)$ & $16(28 \%)$ \\
\hline & $50-59$ & $23(40 \%)$ & $18(31 \%)$ \\
\hline & $60-69$ & $14(25 \%)$ & $6(11 \%)$ \\
\hline & $70+$ & $4(7 \%)$ & $4(7 \%)$ \\
\hline \multirow[t]{7}{*}{ Relationship to patient } & Spouse & & 22 (38\%) \\
\hline & Parent & & $11(19 \%)$ \\
\hline & Child & & $12(21 \%)$ \\
\hline & Sister & & $5(9 \%)$ \\
\hline & Aunt / Uncle & & $1(2 \%)$ \\
\hline & Niece / Nephew & & $2(4 \%)$ \\
\hline & Other & & $4(7 \%)$ \\
\hline \multirow[t]{3}{*}{ Monthly income of household } & $0-R 1000$ & & $52(91 \%)$ \\
\hline & R1001 - R2000 & & $1(2 \%)$ \\
\hline & R2001 - R3000 & & $4(7 \%)$ \\
\hline
\end{tabular}


Swallowing problems did not show a statistical relationship with strain $(p=0.3)$, but clinically four of the five caregivers of patients with swallowing problems scored seven or higher on the CSI. The severe impact of swallowing problems was highlighted in the qualitative data: "... the feeding is a nightmare. She eats so slowly it takes more than an hour... the greatest nightmare is the fear that she will choke" (50 year old, mother).

\section{Caregiver's health status}

The most commonly encountered medical condition reported by caregivers was hypertension from which 23 (40\%) suffered. A further eight (14\%) caregivers had diabetes mellitus and $13(23 \%)$ experienced nervous stress. Other conditions encountered included back problems, cardiac problems, arthritis, asthma and severe migraine. None of the health problems experienced by caregivers had a significant impact on strain except for nervous stress $(\mathrm{p}=0.01)$. In support of this finding emotional adjustment was the CSI question to which the highest number of caregivers

Table 2: Unresolved effects of the stroke

\begin{tabular}{|l|l|l|}
\hline Limitation & Number of Patients & $\begin{array}{l}\text { Statistical relation- } \\
\text { ship with Caregiver } \\
\text { strain (p value) }\end{array}$ \\
\hline $\begin{array}{l}\text { Physically dependent } \\
\text { (<61 on Bl) }\end{array}$ & $20(35 \%)$ & 0.0001 \\
\hline Communication problems & $36(63 \%)$ & 0.6 \\
\hline Swallowing problems & $5(9 \%)$ & 0.3 \\
\hline Personality changes & $42(74 \%)$ & 0.01 \\
\hline $\begin{array}{l}\text { Cognitive and perceptual } \\
\text { problems }\end{array}$ & $14(25 \%)$ & 0.01 \\
\hline Memory problems & $9(16 \%)$ & 0.78 \\
\hline
\end{tabular}

Table 3: Caregivers experiences/involvement during patient stay at WCRC

\begin{tabular}{|l|l|}
\hline & Number of yes responses \\
\hline Felt welcome with admission & $55(96 \%)$ \\
\hline Programme explained to them & $44(77 \%)$ \\
\hline Understood their role in team & $35(61 \%)$ \\
\hline Felt free to ask questions / share fears & $38(67 \%)$ \\
\hline Felt supported & $32(56 \%)$ \\
\hline Felt included in team & $32(56 \%)$ \\
\hline Trained before first weekend home & $31(54 \%)$ \\
\hline Felt their needs were met & $19(33 \%)$ \\
\hline
\end{tabular}

Nineteen $(33 \%)$ caregivers could take time off regularly. The rest $(17 ; 30 \%)$ could take time off when they needed it. Regular time off significantly reduced caregiver strain $(\mathrm{p}=0.03)$.

\section{Caregiver education and knowledge about stroke}

A health care worker explained the diagnosis to just more than half of the caregivers $(33,58 \%)$. Of these $22(39 \%)$ received the explanation from the attending doctor at the time of the stroke. Other health care workers who provided explanations were nurses, physiotherapists and ward doctors at WCRC. Forty eight (84\%) caregivers only partially understood or did not understand the explanation at all. No statistical significant correlation was found between caregiver strain and knowledge of stroke $(\mathrm{p}=0.12)$. However, those who did not understood or only partially understood what a stroke is had a mean caregiver strain score of 7.7, while those who did understood what it is had a mean score of 5.5.

Thirteen $(23 \%)$ caregivers indicated 'other' as their source of information on stroke. They said family members, neighbours, the internet and friends provided information. However, these sources could not be specific as strokes vary widely in its severity and functions affected. Generalised information is not sufficient: "...look for information on stroke on the Internet but the information was too much and scary... we needed information relevant to my husband's stroke" (54 year old, wife).

\section{Caregiver inclusion in the rehabilitation team at WCRC}

Caregivers were positive about the services provided at WCRC: "This rehabilitation centre and the services is a cut above any other facility. It is of world class standard and the staff was very friendly and helpful" (50 year old, wife).

Table 3 provides information on the extent of caregiver involvement in rehabilitation process.

Those caregivers who felt included by the rehabilitation team experienced statistically significantly more strain than those who did not feel included $(p=0.03)$. No other aspect of caregiver involvement in the rehabilitation process showed a significant relationship with caregiver strain. 


\section{Caregiver needs on discharge and in the community}

Caregivers expressed a need for followup and support after the patient is discharged: "... We felt so alone when my brother was discharged home" (38 year old, sister)

"... home visit... should have happened... when she was discharged" (50 year old, mother).

At the time of discharge from WCRC $36(63 \%)$ patients were not referred for follow-up services in the community because there were no appropriate services in their immediate vicinity and no transport was available to access services in adjacent communities. Of the 21 people referred eight (38\%) found the services to be inadequate. Thus in total $44(77 \%)$ caregivers did not receive adequate support after discharge. The community support services that caregivers utilised after the patients were discharged showed no statistical significance on the caregiver strain $(p=0.33)$. The qualitative data indicated that caregivers experience a need for services in the community: "...a place that he could go certain days where he is looked after but also gets therapy" (63 year old, wife). Caregivers suggested adult day care, support groups and peer counselling: “...some support group so that we as caregivers can connect and share our experiences, fears and anxieties. It is good to know there are others in the same boat. It just lightens the burden" (54 year old, wife).

The caregivers further indicated that transport should be provided otherwise it would be difficult to access any community services.

\section{DISCUSSION}

Caregivers, the key players in successful residential integration, are often ignored by health care service providers. In order to ensure successful long term outcomes for patients, it is critical that the rehabilitation team assesses the situation of caregivers and assists them to manage their problems and to identify and build on successes (Pierce et al 2007). Current study findings on inclusion and support of the caregiver during rehabilitation are positive. However, WCRC is a specialised rehabilitation unit and serves as model for the Western Cape Province. Therefore, greater involvement of the caregivers in the general rehabilitation process is a priority.

The results indicate that a higher percentage of caregivers compared to their international counterparts experienced strain (Visser Meiley et al 2005; Rochette et al 2007; Ilsei et al 2008). The caregiver strain was also higher than findings from the study by Kleineibst in 2007 in the same setting, but lower than findings from Wasserman et al (2009). Patients in the Wasserman et al (2009) study received no rehabilitation services and inadequate home based support which might explain the high prevalence of caregiver strain in that population. Patients in the current study received intensive rehabilitation and relatively high levels of caregiver involvement in the rehabilitation process. According to Pierce et al (2007) these are factors that prepare caregivers better for their role and thus decrease the strain. However, these factors did not have a positive impact on strain in the current study. A combination of insufficient education and training, the abrupt break of contact and support on discharge, the lack of support services in the community and financial strain might have negated the effect of rehabilitation.

In order to reduce caregiver stain and ensure more effective, sustainable informal caring, caregiver education and training programmes and health provider follow-up directly post discharge, and long term community support programmes are essential (Schure et al 2006; Pierce et al 2007). Literature indicates that there is often a lack of family education during rehabilitation (Kleineibst, 2007; Hinojosa \& Rittman, 2009). Findings in the current study showed that two rehabilitation team members, namely; the social worker and occupational therapist did not explain the diagnosis to the caregiver, while the ward doctor, nurse and the physiotherapist only explained it to two, three and four caregivers respectively. It is of utmost importance that all professionals repeat and reinforce the explanation regarding a patient's specific condition as a result of the stroke, and expand on it in areas that are relevant to their fields, for example, cognitive function for the occupational therapist and mobility for the physiotherapist.

Training caregivers in the basic skills of moving and handling a patient, activities of daily living and simple nursing tasks can reduce the burden of care, enhance caregiver's quality of life and reduce the risk of injury to caregivers and patients (McCullagh et al 2005; Pierce et al 2007; Hinojosa and Rittman 2009). In the current study the majority of caregivers expressed a need for more training in physical handling skills. This is an area that should be attended to by the relevant professionals as a matter of policy and practice.

Post discharge follow up is necessary to ensure a smooth transition from the rehabilitation facility to the home environment and may impact positively on caregiver anxiety and depression. Home visits can ensure that all environmental barriers have been addressed and that training given in the hospital environment is applicable to the home situation. Grant et al (2002) found that telephonic interventions and problem solving for caregivers of stroke patients after the patient is discharged, improved the wellbeing of the caregiver. This is a less resource intensive way of providing support and intervention and implementation on a trial basis should be considered by management at WCRC.

In accordance with current study findings the literature identified a need for long term support services such as counselling and support groups for caregivers as well as adult day care and respite care for patients. (Kalra et al 2004; Schure et al 2006). Interventions to address emotional experiences of caregivers and the provision of support programmes for caregivers benefit both the caregiver and the patient (Pierce et al 2007).

Support groups can provide the opportunity for reciprocal confiding, information exchange and emotional support (Kalra et al 2004). Both Schure et al (2006) and Kleineibst (2007) found that structured caregiver group support and education programmes reduced strain. However, both these studies look at an intervention with a definite end point and a need for ongoing support was identified in the current study. It is necessary 
for these programmes to take it one step further and guide caregivers to take ownership of support groups in order to ensure sustainability.

Community support programmes in the Western Cape should be supported by the availability of appropriate transport as indicated by participants in the current study and supported by findings from Kleineibst (2007). If not, lack of access will hamper their ability to attend support programmes.

Another grave concern which was highlighted through both quantitative and qualitative data was financial problems. This problem is echoed by studies from the United Kingdom (MacKenzie et al 2007) and Canada (Rochette et al 2007). Similarly Kleineibst (2007) and Wasserman et al (2009) mention financial challenges and changes in employment status as a major cause of caregiver strain. Eleven caregivers in this study had to resign from employment to take on care giving duties. Since economic self-sufficiency of persons with disabilities and their families (Gathiram 2008) should be at the core of rehabilitation programmes one must question why these caregivers were not encouraged by the rehabilitation team to retain their jobs and find an unemployed family member or friend to perform care giving duties.

\section{CONCLUSION}

A high incidence of strain is reported on in the current study. The main contributing factors included financial difficulties, the emotional impact of care giving, physical dependence, personality changes and behaviour problems in the patients as well as high demands on caregiver's time, the confining nature of care giving and a lack community support services. The authors therefore recommend that more emphasis is placed on caregiver education and training, follow up by the rehabilitation team directly post discharge, strengthening of community support services such as support group development, adult day care for stroke survivors, home based care and respite care.

\section{Study limitations}

The study population was limited to one institution in one health district in South
Africa. Therefore the authors caution would be users of these findings and recommendations to carefully consider demographics before extrapolating it to other settings (Pierce et al, 2007).

\section{References}

Gathiram N 2008 A Critical review of the development approach to disability in South Africa. International Journal of Social Welfare 17: $146-155$

Grant JS, Elliot TR, Weaver M, Bartolucci AA, Giger JN 2002 Telephone intervention with family caregivers of stroke survivors after rehabilitation. Stroke 33: 2060-2065.

Hinojosa MS, Rittman M 2009 Association between health education needs and stroke care giver injury. Journal of Aging and Health 21(7): $1040-1058$

Ilsei IB, Feysi H, De Wit L, Putman K and De Weerdt W 2008 Stroke caregivers' strain: prevalence and determinants in the first six months after stroke. Disability and Rehabilitation 30: 523-530

Kalra 1, Evans A, Perez I, Melbourn A, Patel A, Knapp M, Donaldson N 2004 Training carers of stroke patients: Randomised controlled trial. BMJ 328:8.

Kleineibst LJ 2007 The effectiveness of a caregiver support programme to address the needs of primary caregivers of stroke patients in a low socio-economic community. Masters thesis; Department of Physiotherapy. Stellenbosch University

Kroll T, Neri MT, Miller K 2005 Using mixed methods in disability and rehabilitation research. Rehabilitation Nursing 30(3):106-113.

MacKenzie A, Perry L, Lockhart E, Cottee M, Cloud G and Mann H 2007 Family carers of stroke survivors: needs, knowledge, satisfaction and competence in caring. Disability and Rehabilitation 29:111-121

McCullagh E, Brigstocke G, Donaldson N and Kalra L 2005 Determinants of care giving burden and quality of life in caregivers of stroke patients. Stroke 36:2181-2186.

Pierce LL, Steiner V, Govoni A, Thompson TC, Friedemann M 2007 Two Sides to the Caregiving Story. Topics in Stroke Rehabilitation 14(2): 13-20.

Rochette A, Desrosiers J, Bravo G, St-Cyr Tribble D and Bourget A 2007 Changes in Participation Level after Spouse's First Stroke and Relationship to Burden and Depressive Symptoms. Cerebrovascular Diseases 24:255-260
Scherbring M 2002 Effects of care giving perceptions of preparedness on burden in an oncology population. Journal of Gerontology 38:344-348

Schure LM, van den Heuvel ETP, Stewart RE, Sanderman R, de Witte LP, Meyboom-de Jong B 2006 Beyond stroke: Description and evaluation of an effective intervention to support family caregivers of stroke patients. Patient Education and Counselling 62 (2006) 46-55

Uyttenboogaart M, Stewart RE, Vroomen PCAJ, De Keyser J and Luijckx G 2005 Optimizing cut off scores for the Bartel Index and the Modified Rankin Scale for Defining Outcome in Acute Stroke trials. Stroke 36:1984-1987

Visser-Meily A, Post M, Schepers V, Lindeman E 2005 Spouses' quality of life one year after stroke: Prediction at the start of clinical rehabilitation. Cerebrovascular Disease 20:443-448.

Wasserman S, De Villiers L and Bryer A 2009 Community based care of stroke patients in a rural African setting. South African Medical Journal 99:579-583 